\title{
El aprendizaje del álgebra en Educación Secundaria: las estrategias metacognitivas desde la tecnología digital
}

\author{
Algebra learning in Secondary Education: metacognitive strategies from \\ digital technology
}

\author{
Luis Miguel Iglesias Albarrán \\ Doctorando en la Universidad de Alcalá (Educación) \\ IES San Antonio. \\ Bollullos par del Condado, Huelva - España. \\ luismiglesias@gmail.com
}

\begin{abstract}
Isabel Pascual Gómez
Doctora por la Universidad Complutense de Madrid (Departamento MIDE)

Universidad de Alcalá (UAH).

Alcalá de Henares, Madrid - España. isabel.pascualg@uah.es
\end{abstract}

\begin{abstract}
Dianca Arteaga-Martínez
Doctora por la Universidad Complutense de Madrid (Departamento MIDE) Universidad Nacional de Educación a Distancia (UNED).

Madrid - España.

blanca.arteaga@edu.uned.es
\end{abstract}

Resumen: La situación de aprendizaje en las escuelas españolas cambió cuando se decretó el estado de alarma en el mes de marzo de 2020, cerrando las escuelas de una forma brusca. Este artículo muestra la adaptación a un medio de aprendizaje íntegramente digital, llevada a cabo en un instituto de Educación Secundaria, en el sur de España. El trabajo se desarrolla en un aula de Matemáticas con estudiantes de 1415 años, que aprenden conceptos de álgebra. Para ello, se utilizan materiales diversos que facilitan el aprendizaje autónomo y la comunicación docente-estudiante. Los instrumentos de evaluación utilizados son dos plantillas para la resolución de problemas sustentadas en estrategias metacognitivas. Los resultados muestran que los estudiantes han superado los criterios de evaluación marcados para este bloque de contenido, a la vez que el diseño ha facilitado unos niveles de retroalimentación óptima durante todo el proceso de enseñanza-aprendizaje.

Palabras clave: Aprendizaje del algebra. Aprendizaje en línea. COVID-19. Enseñanza virtual. Metacognición. Formación matemática en secundaria.

Abstract: The learning situation in Spanish schools changed when the state of alarm was declared in March 2020, closing schools abruptly. This article shows the adaptation to a fully digital learning environment, carried out in a secondary education center in southern Spain. The study took place in a third-grade algebra class. Various materials were used to facilitate autonomous learning and teacher-student communication. The assessment instruments used were two problem-solving templates based on metacognitive strategies. The results show that the students exceeded the assessment criteria for this block of content, and optimal feedback levels were achieved throughout the teaching and learning process.

Keywords: Learning algebra. Virtual teaching. COVID-19. Online learning. Metacognition. Mathematics secondary education. 


\section{Introducción}

La situación mundial se ha visto afectada por la llegada del coronavirus. Esta pandemia ha supuesto un reto fundamentalmente para los sistemas sanitarios de todos los países, al tiempo que ha planteado un desafío importante para todas las actividades que conllevan interacción entre las personas. En este sentido, el cierre total de las escuelas de manera repentina a comienzos del mes de marzo de 2020 en España trajo consigo el cambio de escenario formativo para millones de estudiantes de todos los niveles educativos. Los edificios de los centros se mantuvieron cerrados, pero la formación continuó desarrollándose hasta el final del curso académico.

La enseñanza cambió de un día para otro en todos los niveles educativos y los docentes tuvieron que trabajar con celeridad para transformar las clases presenciales en clases virtuales. En pocos días, los equipos docentes de los centros tuvieron que poner en marcha una serie de medidas organizativas individualizadas, centradas en la atención tutorial, la realización de adaptaciones metodológicas y curriculares y el seguimiento de los progresos del alumnado. La educación obligatoria tuvo que adaptarse al contexto de manera abrupta y, sin posibilidad de ensayo, abandonar las aulas para atender en los hogares a estudiantes de edades tempranas, con poca autonomía en el aprendizaje.

El primer cambio que hubo que abordar en este proceso fueron las estrategias de comunicación entre docentes y estudiantes. Los docentes construyeron aulas en línea teniendo como primer objetivo evitar en los estudiantes sentimientos de aislamiento; la comunicación se estableció a través de discusiones escritas y el uso de audios o vídeos (TANIS, 2020). En segundo lugar, fue necesario un planteamiento diferencial en cuanto a la tipología de los materiales didácticos que se iban a emplear. Son estos materiales lo relevante en este modelo de enseñanza a distancia, "el material didáctico y las actividades incorporadas a un aula virtual tienen que propiciar un proceso de aprendizaje activo por parte del alumnado" (AREA; ADELL, 2009, p. 404). Y, por último, supuso un cambio en la evaluación de los aprendizajes, que requerían de una rápida retroalimentación. En la revisión sistemática de investigaciones previas que realiza Dorrego (2016) centrada en la evaluación del aprendizaje a distancia, destacamos la que conlleva una retroalimentación orientada a reforzar la motivación y mejorar las estrategias de aprendizaje de los estudiantes. En algunas materias, como la que nos ocupa, supuso un reto adicional por el alto nivel de demanda cognitiva que requieren algunas de sus actividades. De acuerdo con la clasificación de Stein, Grover y Henningsen (1996), las tareas matemáticas se distinguen por el nivel de demanda cognitiva en dos tipos: alto y bajo nivel; en el nivel alto se encuentran aquellas en que se llevan a 
cabo procedimientos que requieren establecer conexiones necesarias para el aprendizaje del algebra en este nivel. El aprendizaje del álgebra presenta mayores dificultades en comparación con otros bloques de contenido de las matemáticas (BOULTON-LEWIS; COOPER; ATWEH; PILLAY; WILLS, 2001), además de que puede considerase como un bloque de contenidos crucial, facilitador de aprendizajes posteriores de mayor nivel de abstracción (NCTM, 2009), por lo que la guía del docente puede resultar fundamental para garantizar el éxito.

Este trabajo muestra los resultados de una intervención en un instituto de Educación Secundaria que pretende dar respuesta a los cambios mencionados, durante el periodo de confinamiento. Se presenta el diseño, implementación y evaluación del proceso de enseñanzaaprendizaje de los contenidos algebraicos correspondientes al tercer curso, utilizando recursos didácticos en un entorno formativo en línea que facilitan un proceso metacognitivo en los estudiantes. El objetivo general es la descripción de la intervención mencionada, para determinar si un ambiente de aprendizaje mediado por tecnología con soportes metacognitivos es eficiente en el aprendizaje del razonamiento algebraico en el nivel de Educación Secundaria. Y como objetivos específicos, la descripción de los cambios que se han producido en una experiencia desarrollada en un momento particular de enseñanza a distancia forzado por el COVID-19:

- Describir las estrategias de comunicación que se establecieron desde el entorno digital para facilitar el aprendizaje.

- Presentar los materiales que se utilizaron desde la perspectiva metacognitiva y la tecnología digital para el aprendizaje del álgebra.

- Evaluar las tareas de los estudiantes utilizando un instrumento metacognitivo.

- Comparar los resultados utilizando dos plantillas con distinto patrón de guía para el estudiante.

\section{Tecnología y metacognición para aprender álgebra}

En un contexto globalizado como el que nos ha tocado vivir, casi transcurrido ya el primer cuarto del siglo XXI, se hace imprescindible un aprendizaje significativo, utilitario y funcional, para interpretar, con ayuda de las matemáticas, el complejo mundo que nos rodea. Así lo revindican los distintos marcos de evaluación y estándares internacionales (TIMSS, PISA) y las distintas evaluaciones de diagnóstico llevadas a cabo por las Agencias de Evaluación Educativas en las comunidades autónomas españolas. Este aprendizaje funcional y utilitario (competencial) de las matemáticas requiere de la introducción de nuevas maneras de enseñar y aprender, que vayan más 
allá del dominio de algoritmos y su trasvase a la resolución de problemas verbales descontextualizados, para pasar a trabajar tareas y situaciones problemáticas abiertas y contextualizadas, que permitan conectar el aula con el mundo real, colocando al estudiante en el centro del proceso de enseñanza-aprendizaje, enriqueciendo este proceso con tecnología.

Según Iglesias (2012, p. 46) la situación actual en el ámbito escolar resulta un tanto paradójica, considerando que es la que es y no puede ser modificada:

Las tecnologías digitales, causantes principales de que la sociedad evolucione a un ritmo tan acelerado y las mismas que piden a gritos cambios en la práctica educativa para formar ciudadanos alfabetizados con las características que demanda la nueva era, son al mismo tiempo las que proporcionan las claves para la solución de bastantes de los problemas educativos actuales.

Señala que la integración de las Tecnologías de la Información y la Comunicación (TIC) en la escuela no es ni de lejos trivial, pero no hay más remedio que acometerla, yendo con cautela porque tecnología sin pedagogía corre el riesgo de convertirse en un manejo mecánico. Esta inclusión de las TIC ha de realizarse impregnada de pedagogía. Ello implica que TIC y Tecnologías del Aprendizaje y del Conocimiento (TAC) deben entrar de manera conjunta en la escuela. No se trata de incidir única y exclusivamente en el aprendizaje de la tecnología. El cambio verdaderamente importante y sustancial radica y se basa en ser capaces de aprender, no ya el uso de la propia tecnología sino, con la tecnología. En definitiva, se trata de colocar las TIC al servicio de una mejora de los procesos de enseñanza aprendizaje (EA) de los contenidos matemáticos, que nos permitan diseñar con y para nuestro alumnado escenarios de aprendizaje personalizados, acercando la escuela a los avances de los entornos tecnológicos actuales. Las TIC deben considerarse como medio para lograr alcanzar objetivos escolares, nunca como un fin. En este sentido, Santos (1996) afirma que es esencial darle más atención a la profundidad del estudio que a la extensión o variedad del contenido ya que la idea de profundidad tiene que ver con el entender los principios centrales de la disciplina, que permiten organizar y tener acceso a otros aspectos, queriendo resaltar la idea que es importante atender el aprendizaje profundo de determinados aspectos de la disciplina y no solamente recorrer superficialmente todo el contenido. El aprendizaje a nivel profundo de algunos aspectos de la disciplina ayudará a los estudiantes a apreciar el potencial de las habilidades y estrategias que aprendan, y les posibilitará usarlas en el estudio de otros contenidos matemáticos y de otras áreas. La tecnología ofrece el potencial de ayudar al estudiante a profundizar en su aprendizaje y es vista como un gran apoyo en su proceso de aprendizaje, en ningún caso sustituyendo al docente sino complementando su acción docente. El estudiante al utilizar software o calculadora debe entender los principios matemáticos que emergen de una determinada 
exploración, así como tener presente que muchas veces la tecnología puede dar una representación gráfica incompleta, u ofrecer únicamente una solución parcial al problema objeto de estudio, que debe ser completada e interpretada por él mismo. Además, este entorno de aprendizaje de las matemáticas mediado por la tecnología puede facilitar un aprendizaje en niveles obligatorios basados en la investigación "buscando un mejor equilibrio entre las dimensiones deductiva y experimental de esta disciplina en su enseñanza" (ARTIGUE, 2015, p.17).

El escenario causado por la pandemia y la necesidad de contar con un modelo de enseñanza aprendizaje completamente dependiente de la tecnología nos ha hecho tomar más conciencia de la importancia de las TIC y la Competencia Digital (estudiantes, docentes y sociedad en general).

Los desarrollos digitales están transformando la manera en que las personas interactúan y abren nuevas rutas para aprender y construir conocimiento matemático, por ejemplo, el desarrollado por los estudiantes a través del uso de las TIC y cómo pueden los maestros utilizar varias herramientas digitales para diseñar e implementar material interactivo para que los estudiantes desarrollen competencias para resolver problemas (SANTOS, 2018).

$\mathrm{Al}$ incorporar las TIC al aprendizaje, el estudiante se encuentra inmerso en un ambiente donde aprender de manera autónoma supone un valor añadido, donde deberá desarrollar su capacidad de autorregulación y ser capaz de aprender a aprender. Siguiendo a Mayor (1991 citado en ALLUEVA, 2002), se hace imprescindible por tanto trabajar con especial énfasis las estrategias de aprendizaje metacognitivas, en combinación con las cognitivas y orécticas, tanto las relativas al desarrollo del proceso de aprendizaje (planteamiento, seguimiento y evaluación) como la actividad reflexiva del sujeto que se produce en la toma de conciencia y en control. La metacognición es un componente del aprendizaje especialmente relevante puesto que en la medida en que el estudiante adquiera las habilidades metacognitivas, el proceso de aprendizaje será más satisfactorio. La resolución de problemas y la metacognición son dos términos muy ligados desde la década de 1980, que se relacionan desde tres aspectos fundamentales: autorregulación, monitorización y control. "La metacognición, las creencias y las prácticas matemáticas se consideran aspectos críticos del pensamiento matemático. Pero hay más, la persona que piensa matemáticamente tiene una manera particular de ver el mundo, de representarlo, de analizarlo" (SCHOENFELD, 1992, p. 81).

La resolución de problemas antes mencionada ha de encaminarse hacia elementos globales que quedan recogidos en la competencia matemática, a través de situaciones escolares que ubiquen al estudiante en tareas de la vida cotidiana. Son distintos los términos utilizados para nombrar a este tipo de tareas, nos quedamos con el acuñado por Palm (2008) como "tareas auténticas", al referirnos a problemas establecidos como tareas reales que dan lugar a situaciones reales y cercanas 
al estudiante, "más allá de saber resolver de forma mecánica ejercicios descontextualizados, a través de 'fórmulas' que se aplican, pero no se comprenden” (ALSINA; VÁSQUEZ-ORTIZ, 2016, p. 43).

Si pretendemos orientar los aprendizajes de los estudiantes a la resolución de tareas auténticas se hace necesario también potenciar y aumentar su nivel de razonamiento algebraico ya que éste se antoja clave (MECD, 2012), desde el punto de vista matemático, para modelar el cambio y las relaciones con las funciones y ecuaciones pertinentes, además de para crear, interpretar y traducir las representaciones simbólicas y gráficas de las relaciones. Esta necesidad de modelizar matemáticamente las relaciones en lo cotidiano, proceso definido en el marco de PISA como matematización horizontal, entre otras, ha propiciado que en las últimas décadas, el desarrollo del razonamiento algebraico se haya erigido como un factor crítico, de especial relevancia para el éxito en matemáticas, porque proporciona a los estudiantes la capacidad de transición desde el aprendizaje empleando únicamente números y las operaciones aritméticas básicas para comprender, a posibilitarles hacer uso de conceptos matemáticos más complejos para poder explicar e interpretar la realidad (KAPUT, 2000). Según Rabino y Cuello (2017), por lo general, el tratamiento del álgebra se inicia en el primer curso de educación secundaria en forma abstracta otorgando mayor importancia a los procesos de resolución de ecuaciones que al significado, imponiendo un lenguaje carente de sentido, trabajando a posteriori numerosos ejercicios de repetición mecánicos y problemas donde aplican lo visto en forma de reglas y definiciones, sin establecer relaciones que impliquen la comprensión del problema, ni promover el razonamiento y el pensamiento algebraico de los estudiantes, teniendo como consecuencia esta metodología tradicional de enseñanza del álgebra que los estudiantes rechacen este contenido, al no encontrarle sentido. Otra consecuencia de este tipo de enseñanza del álgebra es que imposibilita que el estudiante tenga el control sobre lo que hace -de un modo mecánico- lo que implica que el más mínimo error le conduce inevitablemente al fracaso. El entrenamiento metacognitivo y el trabajo con contextos reales y situaciones cargadas de significado, más allá de sustituir números por letras, puede contribuir a evitar estas consecuencias.

\footnotetext{
Mevarech y Kramarski (2017, p. 173) se plantean las siguientes cuestiones: ¿Cuáles son los problemas matemáticos y las habilidades útiles para desarrollar ciudadanos competentes en las sociedades impulsadas por la innovación? y ¿qué métodos son efectivos para promover estas habilidades, acaso existe evidencia de que ciertas pedagogías funcionan realmente en nuestros sistemas educativos altamente estructurados?
}

Llegando a la conclusión de que, si bien ninguna de las dos tiene respuesta cerrada, ni son triviales, en la construcción de potenciales respuestas a ambas se encontraría incluida la 
metacognición. En el primer caso porque existe un amplio consenso en que, además de aprender a resolver los problemas matemáticos tradicionales, los estudiantes deben familiarizarse con las capacidades, habilidades y procesos necesarios para resolver problemas desconocidos, complejos y no rutinarios, para lo que se requiere potenciar el aprender a aprender. Respecto a la segunda pregunta, la metacognición haría de mediadora entre los defensores de los métodos de enseñanza tradicionales, que propugnan volver a las habilidades básicas, y aquellos que apuestan por la necesidad de poner en práctica pedagogías basadas en la indagación, para la mejora de la solución de tareas y problemas complejos no rutinarios. La metacognición aportaría métodos de enseñanza basados en la evidencia que fusionarían, de una parte, el hacer hincapié en la fluidez del uso de los algoritmos y, de otra parte, la comprensión conceptual, el razonamiento, la creatividad, la resolución de problemas y la comunicación en matemáticas. Potenciar todo ello mediante estrategias metacognitivas contribuiría a consolidar en los estudiantes un aprendizaje matemático más sólido y significativo, el cual no podrían obtener únicamente realizando actividades y problemas rutinarios basados en la repetición de algoritmos.

Continuando con la revisión de conocimientos, cabe destacar la tesis de Gama (2004), en la que se realiza la integración de instrucciones metacognitivas en un entorno de aprendizaje interactivo sustentada en estudios empíricos que han demostrado que los estudiantes con un buen desarrollo de conciencia cognitiva obtienen mejores resultados que aquellos con un menor nivel. Las teorías de la metacognición destacan la importancia de la autorreflexión como un medio para mejorar la capacidad de uno mismo, para monitorear, autodirigir y evaluar los propios procesos de aprendizaje. En dicho trabajo se analiza la intención de incluir instrucciones metacognitivas en entornos de aprendizaje interactivos, pero se indica que, en la práctica, es muy difícil de implementar, porque es muy complicado proporcionar orientación adecuada sobre la metacognición a los estudiantes.

Al hilo del impacto del entrenamiento metacognitivo sobre el razonamiento algebraico de los estudiantes se ha revisado el trabajo de Lee, Capraro, Capraro y Bicer (2018), consistente en un metaanálisis de 18 investigaciones sobre la mejora del razonamiento algebraico obtenido a través del entrenamiento metacognitivo. En dicha investigación se deduce que se puede explicar la importancia de la metacognición para el razonamiento algebraico. Se analizaron los diferentes tipos de entrenamiento metacognitivos examinados en los estudios incluidos (uso de ordenadores, capacitación online, individual y colaborativa, y autorregulación, entre otros). Independientemente del tipo de método, se concluyó que el entrenamiento metacognitivo ayuda a los estudiantes a desarrollar su razonamiento algebraico. Estar expuesto a estrategias metacognitivas podría explicar 
la mejora del razonamiento algebraico de los estudiantes. Sin embargo, se observó que el grado de mejora en el razonamiento algebraico puede variar según el tipo de método de entrenamiento metacognitivo empleado. Se podría estudiar a futuro qué tipo de entrenamiento tiene un mayor impacto en el razonamiento algebraico de los estudiantes.

\section{Descripción de la experiencia}

Esta investigación, fundamentada en la revisión teórica expuesta de manera previa, fue realizada durante los meses marzo y abril del curso 2019-20 en un Instituto de Educación Secundaria (IES) público de Andalucía (España). Los indicadores de la Consejería de Educación sitúan el Índice Socioeconómico y Cultural del centro en el tramo 6, siendo 10 el tramo mayor. El centro tiene cinco líneas en el primer y segundo curso, y cuatro en tercero y cuarto de Educación Secundaria.

\subsection{Participantes}

La muestra está integrada por un grupo de 27 estudiantes (13 niñas y 14 niños), con edades comprendidas entre los 14 y 15 años, bastante homogéneo en cuanto a rendimiento educativo. Pertenecen a un grupo cuyo itinerario son las matemáticas académicas. El nivel detectado en la prueba inicial de comienzo de curso es medio; muestran un buen comportamiento en clase, motivación y actitud favorable hacia el aprendizaje. En el primer trimestre del curso todos los estudiantes del grupo adquirieron de manera satisfactoria los objetivos fijados para la asignatura de matemáticas.

\subsection{Contenidos y criterios de evaluación}

Los contenidos trabajados se sitúan en el bloque de álgebra de la asignatura Matemáticas Orientadas a las Enseñanzas Académicas (expresiones algebraicas, igualdades notables y operaciones con polinomios, ecuaciones de primer grado complejas con paréntesis y denominadores, ecuaciones de segundo grado, sistemas de dos ecuaciones lineales con dos incógnitas y resolución de problemas, con ecuaciones y sistemas).

Los criterios de evaluación relacionados con el álgebra (Tabla 1) se ajustan a lo que la legislación señala para el nivel educativo (España, 2016). 
Tabla 1 - Criterios de evaluación para el bloque de contenido relativo al álgebra (bloque 2. números y álgebra - matemáticas académicas $3^{\circ} \mathrm{ESO}$ )

\begin{tabular}{|l|l|l|l|l}
$\begin{array}{l}\text { Criterio de } \\
\text { evaluación }\end{array}$ & $\begin{array}{l}\text { Descripción } \\
\mathbf{2}\end{array}$ & $\begin{array}{l}\text { Obtener y manipular expresiones simbólicas que describan sucesiones } \\
\text { numéricas, observando regularidades en casos sencillos que incluyan } \\
\text { patrones recursivos. }\end{array}$ \\
\hline $\mathbf{3}$ & $\begin{array}{l}\text { Utilizar el lenguaje algebraico para expresar una propiedad o relación dada } \\
\text { mediante un enunciado, extrayendo la información relevante y } \\
\text { transformándola. }\end{array}$ \\
\hline 4 & $\begin{array}{l}\text { Resolver problemas de la vida cotidiana en los que se precise el } \\
\text { planteamiento y resolución de ecuaciones de primer y segundo grado, } \\
\text { ecuaciones sencillas de grado mayor que dos y sistemas de dos ecuaciones } \\
\text { lineales con dos incógnitas, aplicando técnicas de manipulación algebraicas, } \\
\text { gráficas o recursos tecnológicos, valorando y contrastando los resultados } \\
\text { obtenidos. }\end{array}$ \\
\hline
\end{tabular}

Fuente: España (2016, p.193)

\subsection{Metodología docente y descripción de los materiales}

De acuerdo con las recomendaciones para el diseño de las actividades en línea, se han diseñado con un lenguaje sencillo, adaptado a las capacidades de los estudiantes, teniendo en cuenta los conocimientos previos de los estudiantes, con tareas auténticas y problemas relacionados con la vida real, sus experiencias e intereses. Al particularizar en los contenidos de álgebra se han seguido los principios del programa IA (Intensified Algebra I; MARTINEZ; BRAGELMAN; STOELINGA, 2016, p. 8), donde se secuencia "para que los estudiantes primero desarrollen conceptos fundamentales trabajando con contexto representaciones tempranas de la unidad, y luego progresar a procedimientos más abstractos y simbólicos de la unidad, cuando pueden ser construidos sobre una base conceptual establecida".

Respecto a los materiales, el libro de texto en formato papel ha sido reemplazado por materiales guiados interactivos y baterías de actividades aleatorias autocorregibles, con retroalimentación inmediata, en aras de favorecer el aprendizaje autónomo y la autorregulación de los estudiantes. Los materiales se han configurado de manera que su resolución facilite al docente 


\section{Dialogia}

ALBARRÁN, Luis Miguel Iglesias; GÓMEZ, Isabel Pascual; ARTEAGA-MARTÍNEZ, Blanca. El aprendizaje del álgebra en Educación Secundaria: las estrategias metacognitivas desde la tecnología digital

la detección de los obstáculos de aprendizaje en sus estudiantes (Figura 1), evite el abandono de la materia, y que se aprendan de manera errónea algunos conceptos y procedimientos.

Figura 1 - Muestra de trabajo de estudiante. Reflexión

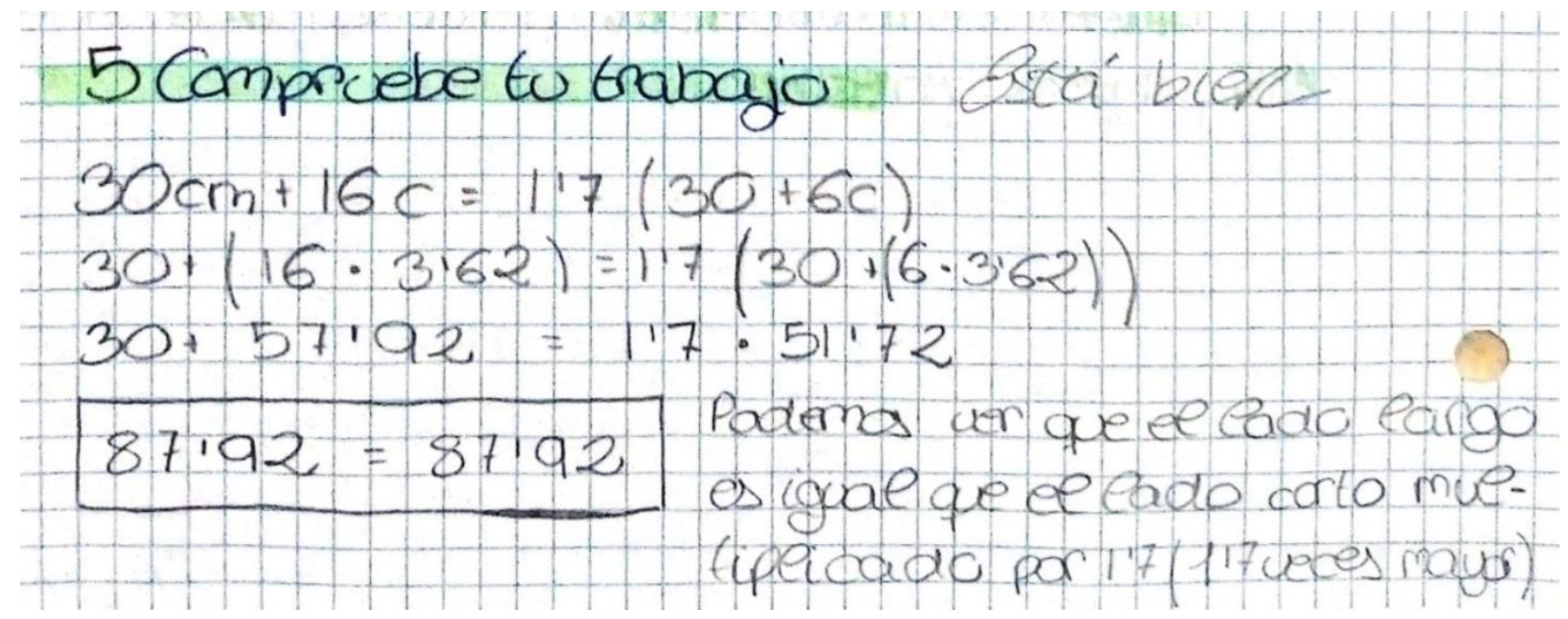

Fuente: Estudiante \#16

Las tareas solicitadas, ricas y creativas, buscaban el aumento del nivel de exigencia para los estudiantes desde el punto de vista cognitivo, adaptadas al contexto de enseñanza a distancia. Sirvan como ejemplo los cuestionarios online diseñados ad hoc, usados de manera minoritaria y puntual; el diseño de actividades y problemas abiertos con varias posibles soluciones (Figura 2); la creación de tareas aleatorias diferentes para cada estudiante; los materiales multimedia elaborados por los estudiantes con problemas de la vida cotidiana, resueltos con ecuaciones y sistemas (explicando su resolución paso a paso); y la resolución de puzles o de laberintos de ecuaciones. Cabe señalar que parte de los materiales que se utilizaron no fueron elaborados por el docente, sino que se utilizaron materiales de repositorios virtuales como los incluidos en el Proyecto Descartes (https://proyectodescartes.org/descartescms/). 


\section{Dialogia}

ALBARRÁN, Luis Miguel Iglesias; GÓMEZ, Isabel Pascual; ARTEAGA-MARTÍNEZ, Blanca. El aprendizaje del álgebra en Educación Secundaria: las estrategias metacognitivas desde la tecnología digital

Figura 2 - Ejemplo de tarea abierta tipo Open Middle en geogebra

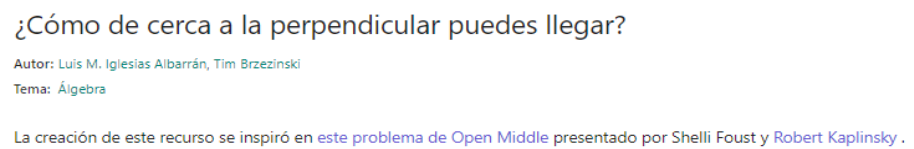

TAREA 1. OBLIGATORIA. INSTRUCCIONES:

Usando los dígitos 1-9 como máximo una vez, cada uno completa los coeficientes de las tres ecuaciones lineales a la derecha para que dos líneas sean paralelas y la tercera línea sea una transversal que pase por las otras dos.

Nota: ¡Hay varias posibilidades!

TAREA 2. VOLUNTARIA. INSTRUCCIONES:

Repita la tarea anterior, pero esta vez intente obtener la transversal (no paralela a las otras 2 líneas) tan "cerca de perpendicular" a las otras dos líneas paralelas como sea posible.

¿Qué tan cerca de 90 grados puede obtener la transversal para intersecar las otras 2 líneas paralelas?

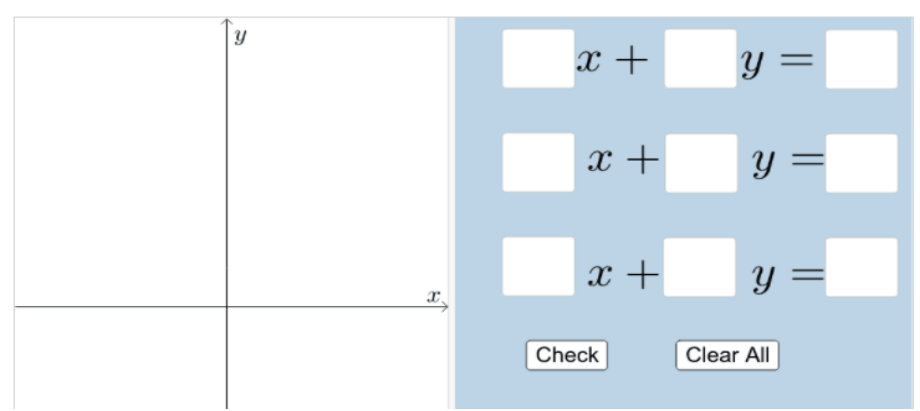

Fuente: Iglesias (2020a)

Para atender a la diversidad, se elaboraron materiales ad hoc adaptados a las características propias del grupo de estudiantes (Figura 3), proporcionando a los estudiantes un camino reflexivo para la resolución de problemas, no penalizando el error, sino utilizándolo como elemento de aprendizaje. Es importante el modo en que los estudiantes perciben cómo el docente trata el error, ya que condicionará las reacciones individuales y la autorregulación de su esfuerzo (STEUER; ROSENTRITT-BRUNN; DRESEL, 2013).

Figura 3 - Muestra de trabajo de estudiante (\#20). Construcción de laberinto de ecuaciones

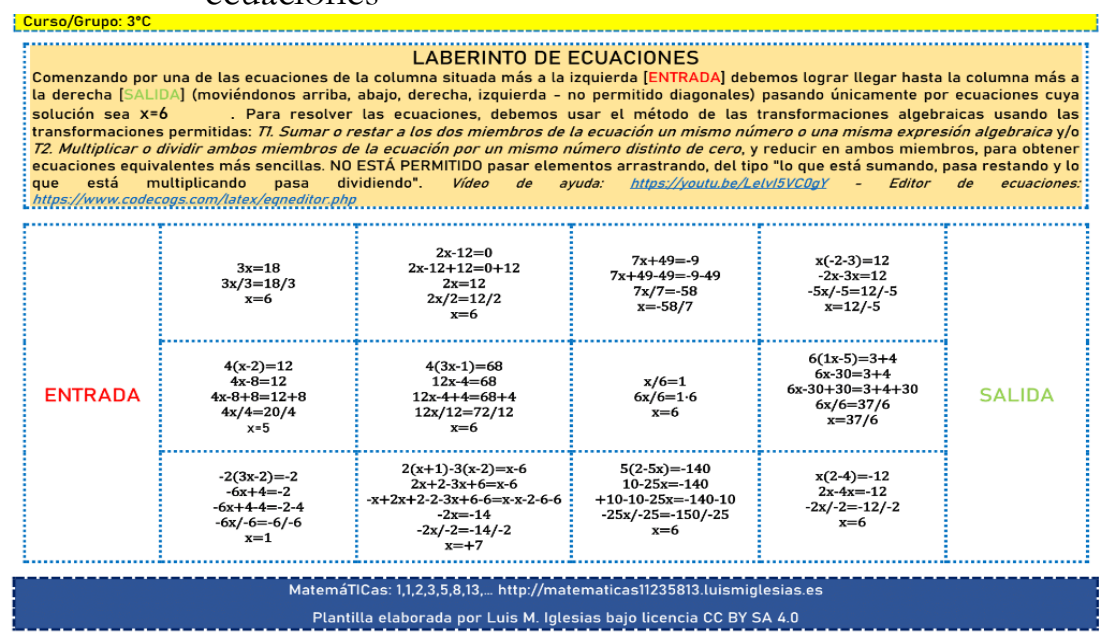

Fuente: Iglesias (2020b). 
Respecto a la comunicación, que señalamos como uno de los cambios fundamentales en el nuevo escenario, se utilizaron estrategias de comunicación asíncrona (mensajes en plataforma, emails, chats en la retroalimentación de las tareas, preparación y grabación de vídeos) combinadas con estrategias de comunicación síncrona (videoconferencias en directo, sesiones expositivas sobre contenidos nuevos y resolución de dudas). El esfuerzo en la selección, elaboración del material y los planes de trabajo semanales para comunicarse con el alumnado y familias a través de los canales telemáticos, ha sido igualmente importante al requerir un trabajo minucioso, con explicaciones claras, concisas y concretas, buscando minimizar ambigüedades, evitar dudas y favorecer el trabajo autónomo de los estudiantes; alternando materiales escritos, que se han digitalizado, con materiales audiovisuales construidos por los estudiantes y el docente. Las sesiones de videoconferencia y resolución de dudas eran fluidas y centradas en los contenidos de mayor complejidad, gracias al trabajo de interacción del alumnado con el material interactivo guiado y utilizado de manera previa, y la lectura atenta de la retroalimentación proporcionada en las distintas actividades y tareas. A todo ello hay que sumar, además, el esfuerzo requerido para superar el obstáculo del lenguaje matemático/algebraico, y plasmarlo por escrito en producciones digitales, que exigen un alto nivel de competencia digital docente y discente.

Para evaluar cada una de estas tareas se construyeron categorías de análisis a partir del análisis de investigaciones previas (WILSON, 1991 citado en FORD, 2018). La clave para la construcción de las categorías de análisis (Tabla 2) radica en la importancia de asignar valores numéricos sobre los distintos focos de observación de la tarea, esto facilitará el análisis e interpretación del proceso y los resultados de los estudiantes. En la situación particular en que nos encontrábamos, fue fundamental contar con ítems concretos (categorías) en el proceso de evaluación, que aportaban al docente información cualitativa del proceso y facilitaban la posterior retroalimentación al estudiante.

Para la construcción de las categorías se utilizó las recogidas en la "Escala analítica para la resolución de problemas" (WILSON, 1991 citado en FORD, 2018), a la que se añadió una categoría más relacionada con la representación pictórica del problema. El contraste entre la teoría existente y el planteamiento específico de las tareas de resolución de problemas dio lugar a la elaboración de una matriz de valoración (con cinco niveles y cuatro categorías por nivel) que facilitó el procedimiento de evaluación y detección de errores particulares en cada uno de los procesos. Esta matriz fue evaluada por tres expertos, especialistas en didáctica de la matemática. 
Tabla 2 - Categorías para el análisis metacognitivo de la tarea matemática

\begin{tabular}{|c|c|c|c|c|}
\hline Puntuación & $\begin{array}{l}\text { Entender el } \\
\text { problema }(\mathrm{E})\end{array}$ & $\begin{array}{l}\text { Visualización } \\
\text { (V) }\end{array}$ & $\begin{array}{l}\text { Resolver el } \\
\text { problema (Rs) }\end{array}$ & $\begin{array}{l}\text { Responder al } \\
\text { problema ( } \mathbf{R p})\end{array}$ \\
\hline 0 & Sin intento. & $\begin{array}{l}\text { No se } \\
\text { proporcionó } \\
\text { ningún diagrama. }\end{array}$ & Sin intento. & $\begin{array}{l}\text { Ninguna respuesta } \\
\text { o respuesta } \\
\text { incorrecta basada } \\
\text { en un plan } \\
\text { inapropiado. }\end{array}$ \\
\hline 1 & $\begin{array}{l}\text { Malinterpreta el } \\
\text { problema } \\
\text { completamente. }\end{array}$ & $\begin{array}{l}\text { El diagrama no } \\
\text { es una } \\
\text { representación } \\
\text { vinculada al } \\
\text { problema } \\
\text { (representa } \\
\text { varios errores de } \\
\text { identificación } \\
\text { importantes). }\end{array}$ & $\begin{array}{l}\text { Plan totalmente } \\
\text { inapropiado. }\end{array}$ & $\begin{array}{l}\text { Respuesta } \\
\text { incorrecta debida } \\
\text { a error de } \\
\text { interpretación de } \\
\text { los datos del } \\
\text { enunciado o error } \\
\text { de cálculo. }\end{array}$ \\
\hline 2 & $\begin{array}{l}\text { Malinterpreta la } \\
\text { mayor parte del } \\
\text { problema. }\end{array}$ & $\begin{array}{l}\text { El diagrama } \\
\text { puede } \\
\text { representar el } \\
\text { problema con } \\
\text { algunos errores } \\
\text { de identificación } \\
\text { importantes. }\end{array}$ & $\begin{array}{l}\text { Procedimiento } \\
\text { parcialmente } \\
\text { correcto pero } \\
\text { con un error } \\
\text { importante. }\end{array}$ & $\begin{array}{l}\text { Respuesta parcial } \\
\text { para el problema. }\end{array}$ \\
\hline 3 & $\begin{array}{l}\text { Malinterpreta una } \\
\text { parte menor del } \\
\text { problema }\end{array}$ & $\begin{array}{l}\text { El diagrama } \\
\text { generalmente } \\
\text { representa el } \\
\text { problema con } \\
\text { pocos errores de } \\
\text { identificación } \\
\text { menores. }\end{array}$ & $\begin{array}{l}\text { Procedimiento } \\
\text { sustancialmente } \\
\text { correcto con } \\
\text { omisión menor o } \\
\text { error de } \\
\text { procedimiento. }\end{array}$ & $\begin{array}{l}\text { Respuesta } \\
\text { etiquetada } \\
\text { incorrectamente. }\end{array}$ \\
\hline 4 & $\begin{array}{l}\text { Comprensión } \\
\text { completa del } \\
\text { problema }\end{array}$ & $\begin{array}{l}\text { El diagrama es } \\
\text { una } \\
\text { representación } \\
\text { válida y } \\
\text { adecuadamente } \\
\text { vinculada del } \\
\text { problema. }\end{array}$ & $\begin{array}{l}\text { Un plan que } \\
\text { podría conducir a } \\
\text { una solución } \\
\text { correcta sin } \\
\text { errores } \\
\text { aritméticos }\end{array}$ & Solución correcta. \\
\hline
\end{tabular}

Fuente: Elaboración propia a partir de Wilson (1991 citado en FORD, 2018).

Estas categorías se utilizan de manera particular en la tarea de álgebra, pero consideramos pueden utilizarse en cualquier tarea matemática.

4 Evaluación del proceso de aprendizaje

Para evaluar la eficacia de esta intervención se utilizó como instrumento (Tabla 3) el propuesto por FORD (2018), con un formato de ficha o plantilla. Este instrumento estaba 
originalmente en inglés y se desarrolló un proceso de adaptación en varias fases mediante la traducción directa e inversa. La primera fase dio lugar a la traducción al castellano de la versión en inglés; en la segunda fase un investigador conocedor de ambos idiomas, que no conocía la versión original, realizó una traducción inversa; ambas versiones se remitieron a un tercer investigador de la didáctica de las matemáticas, con manejo de ambos idiomas para confirmar la equivalencia entre los resultados.

Tabla 3 - Plantillas de resolución de problemas

\section{Plantilla $1 \quad$ Plantilla 2}

1. Lee el problema y reescribe el enunciado con 1 . Lee el problema y reescribe el enunciado con tus propias palabras tus propias palabras

2. Identifica los datos y la información importante

Pregúntate a ti mismo/a. (1) ¿Comprendo el problema? (2) ¿Entiendo lo que significan todas las palabras?

3. Realiza un dibujo o un diagrama que te ayude 2. Identifica los datos y la información a visualizar el problema importante Pregúntate a ti mismo/a. (1) De lo que se

4. Resuelve el problema pregunta, ¿qué es lo que no conozco? (2)

5. Comprueba tu trabajo ¿Cuáles son los datos, qué información puedo extraer del enunciado?

3. Realiza un dibujo o un diagrama que te ayude a visualizar el problema

Pregúntate a ti mismo/a. (1) ¿Puedo conectar los datos y lo que no conozco de manera visual? (2) ¿Es mi dibujo o mi diagrama una buena representación del problema?

4. Resuelve el problema

Pregúntate a ti mismo. (1) ¿Necesito fórmulas, ecuaciones o alguna notación especial? (2) ¿Sé ahora cómo obtener la solución?

5. Comprueba tu trabajo Pregúntate a ti mismo. (1) ¿Los cálculos que he realizado son correctos? (2) ¿Es mi respuesta razonable?

Fuente: Traducido de Ford (2018).

Para cada una de las plantillas se utilizaron dos problemas situados en un contexto de la vida cotidiana. Las tareas matemáticas con un contexto de la vida real facilitan la conexión del nuevo conocimiento con las experiencias personales, a la vez que son capaces de involucrar a los estudiantes en trabajos más exigentes cognitivamente (MALI; GERAMI; ULLAH; MESA, 2019). 


\section{Dialogia}

ALBARRÁN, Luis Miguel Iglesias; GÓMEZ, Isabel Pascual; ARTEAGA-MARTÍNEZ, Blanca. El aprendizaje del álgebra en Educación Secundaria: las estrategias metacognitivas desde la tecnología digital

La diferencia principal entre ambas plantillas es el tipo de pautas y orientaciones que se ofrecen al estudiante para resolver los problemas, más completa y precisa en la segunda plantilla, lo que debería facilitar la tarea metacognitiva.

El diseño de la investigación es descriptivo, utilizando como instrumento para la evaluación las plantillas metacognitivas (FORD, 2018).

\section{Resultados}

En este apartado mostraremos los resultados obtenidos sobre el nivel de dominio de los criterios de evaluación marcados en relación con el manejo y aplicación de los contenidos de álgebra, y las diferencias entre las dos plantillas elaboradas como guía para la resolución de problemas.

Figura 4 - Gráficos de medianas en cada uno de los problemas de acuerdo a las categorías

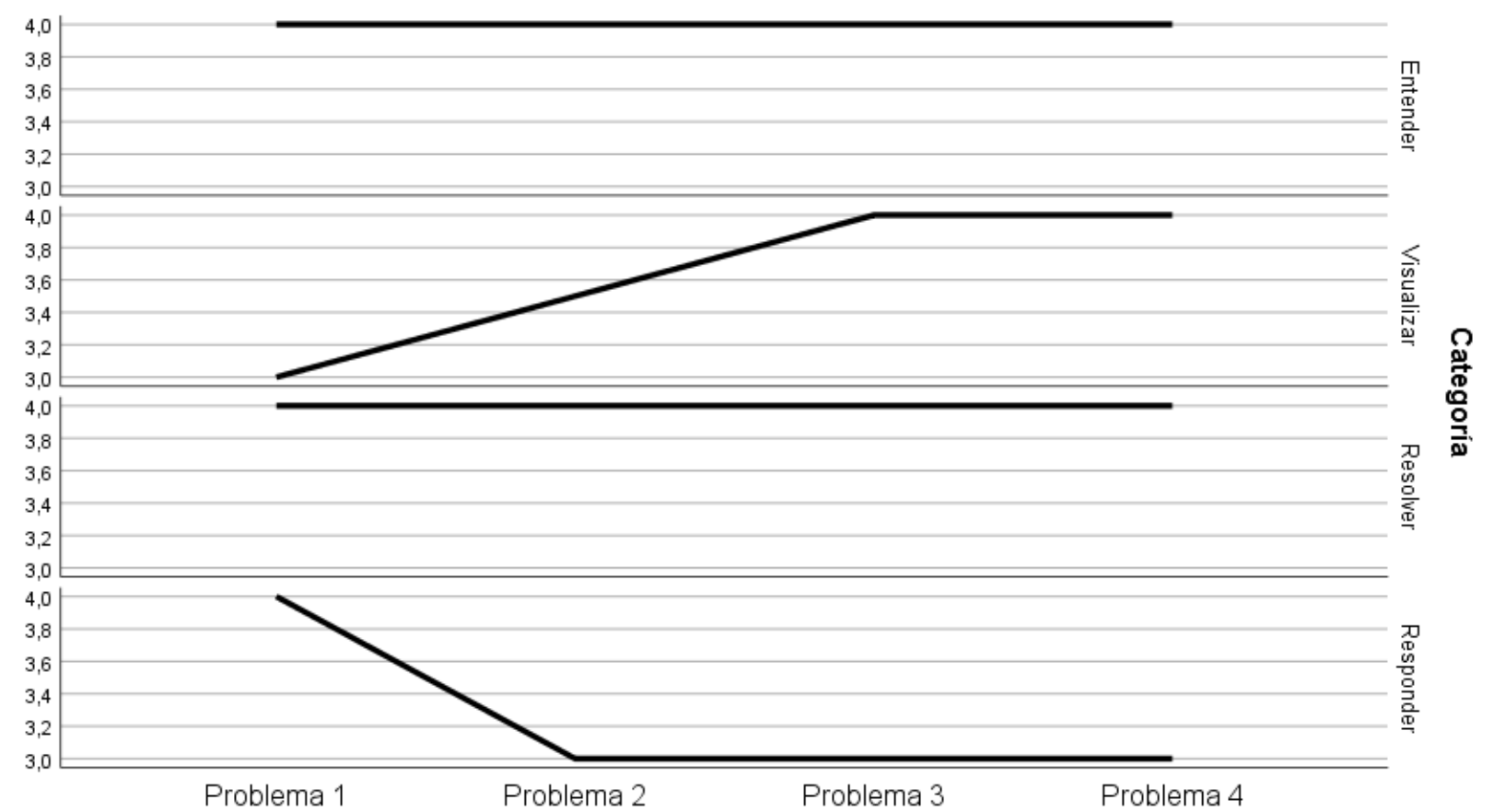

Fuente: Elaboración propia.

De acuerdo a los valores de la mediana (Tabla 4), podemos señalar que se sitúan de manera general en 3 y 4 (Figura 4). Podría afirmarse, por lo tanto, que se ha logrado un buen dominio de los contenidos. Respecto a la comparación entre ambas plantillas se observa una mayor dispersión en los problemas de la Plantilla 1, más concretamente en la categoría "Visualización” de los problemas 1 y 2 . 


\section{Dialogia}

ALBARRÁN, Luis Miguel Iglesias; GÓMEZ, Isabel Pascual; ARTEAGA-MARTÍNEZ, Blanca. El aprendizaje del álgebra en Educación Secundaria: las estrategias metacognitivas desde la tecnología digital

Tabla 4 - Descriptivos de las categorías

\begin{tabular}{lllllllll} 
& $\mathrm{E}(\mathrm{p} 1)$ & $\mathrm{V}(\mathrm{p} 1)$ & $\mathrm{Rs}(\mathrm{p} 1)$ & $\mathrm{Rp}(\mathrm{p} 1)$ & $\mathrm{E}(\mathrm{p} 2)$ & $\mathrm{V}(\mathrm{p} 2)$ & $\mathrm{Rs}(\mathrm{p} 2)$ & $\mathrm{Rp}(\mathrm{p} 2)$ \\
$\mathbf{M d n}$ & 4 & 3 & 4 & 4 & 4 & 3.5 & 4 & 3 \\
\hline Mo & 4 & 4 & 4 & 4 & 4 & 4 & 4 & 3 \\
\hline RI & 1 & 2 & 0 & 1 & 1 & 3 & 0 & 1 \\
\hline \multirow{2}{*}{ Mdn } & $\mathrm{E}(\mathrm{p} 3)$ & $\mathrm{V}(\mathrm{p} 3)$ & $\mathrm{Rs}(\mathrm{p} 3)$ & $\mathrm{Rp}(\mathrm{p} 3)$ & $\mathrm{E}(\mathrm{p} 4)$ & $\mathrm{V}(\mathrm{p} 4)$ & $\mathrm{Rs}(\mathrm{p} 4)$ & $\mathrm{Rp}(\mathrm{p} 4)$ \\
\hline Mo & 4 & 4 & 4 & 3 & 4 & 4 & 4 & 3 \\
\hline RI & 0 & 1 & 4 & 3 & 4 & 4 & 4 & 3 \\
\hline
\end{tabular}

Fuente: Elaboración propia.

$\mathrm{Al}$ analizar algunas de las muestras de trabajo, tenemos oportunidades de retroalimentación específica. Por ejemplo, encontramos como algunos estudiantes realizan representaciones de conceptos aislados que no ayudan a la comprensión específica del problema, mientras que otros realizan dibujos sencillos pero que facilitan la visualización de los datos (Figura 5). 


\section{Dialogia}

ALBARRÁN, Luis Miguel Iglesias; GÓMEZ, Isabel Pascual; ARTEAGA-MARTÍNEZ, Blanca. El aprendizaje del álgebra en Educación Secundaria: las estrategias metacognitivas desde la tecnología digital

Figura 5 - Muestra de trabajo de tres estudiantes. Representación

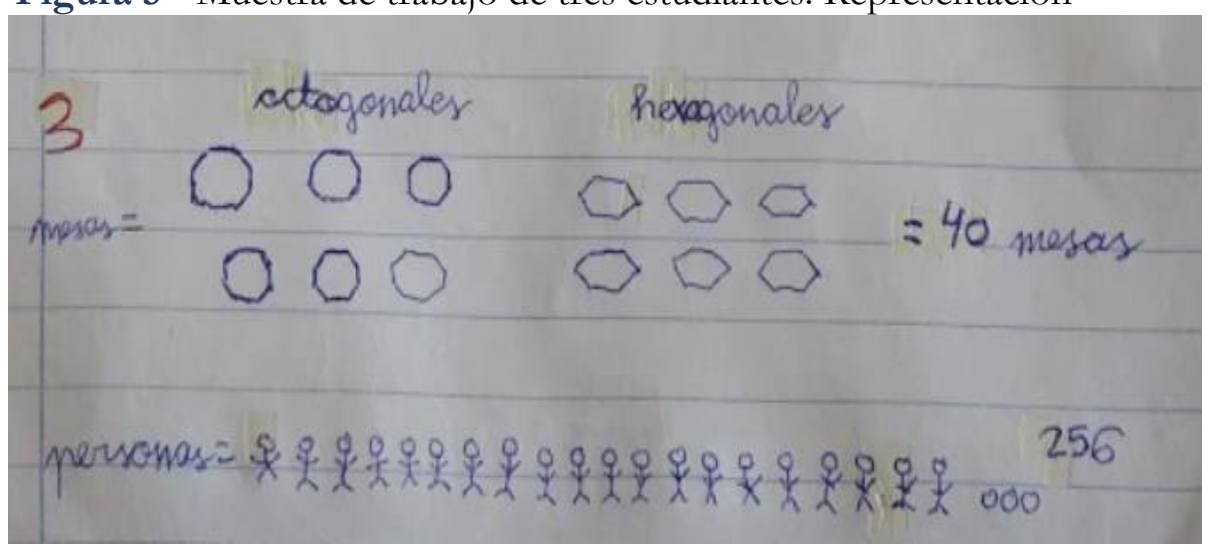

3 Realiza un dibujo o un diagrama que te ayude a visualizar el problema.

Reguntate a timismo/a. (1) ¿lledo conectar eos datos y es que no canozco de marena ui sual? (2) ¿Es mi dibujo o mi diagrama una buena representación del problema?
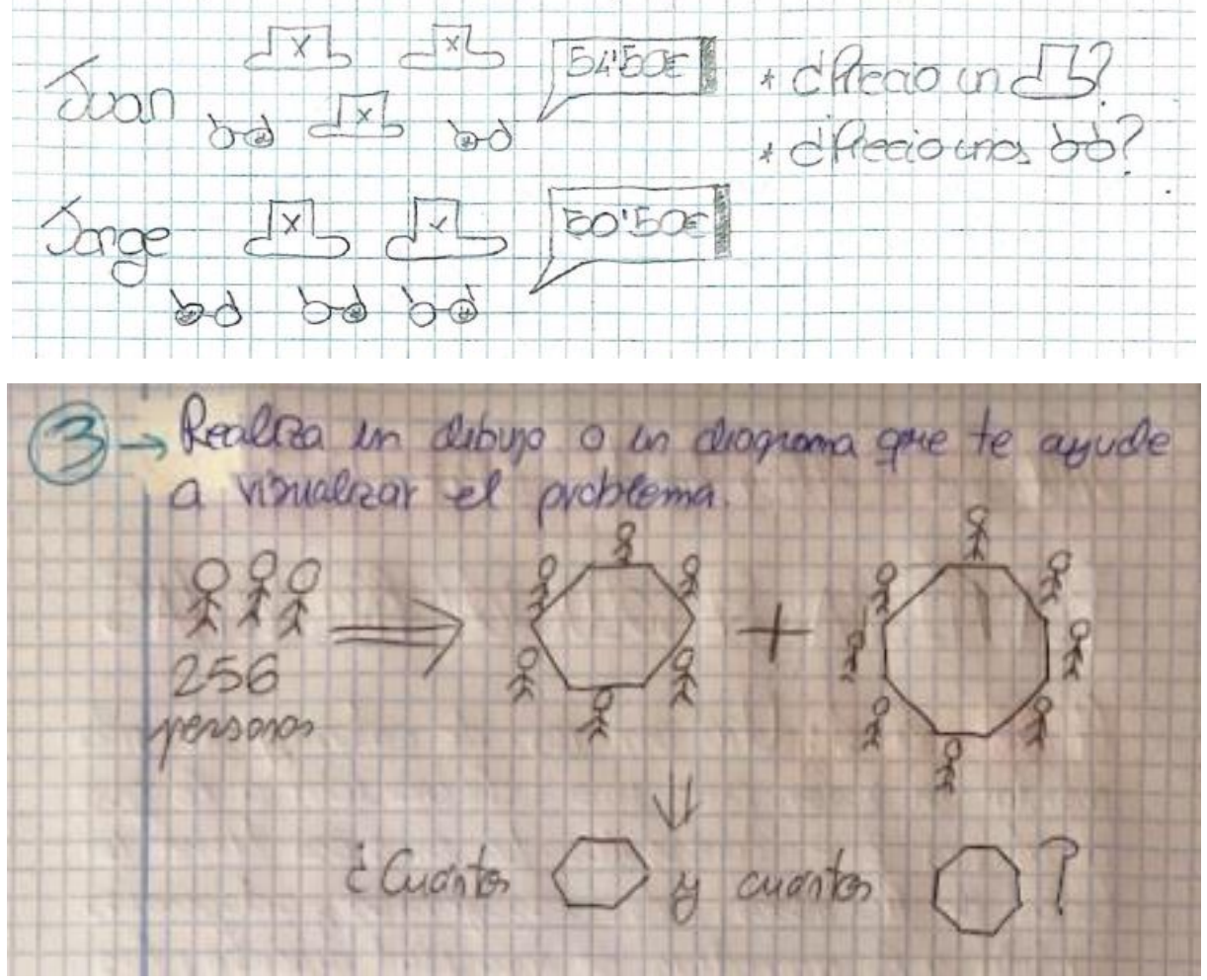

Fuente: Estudiantes \#14, \#16 y \#21.

El trabajo realizado de manera previa a estas plantillas ha facilitado una estructuración en las respuestas (Figura 6), y un nivel alto de escritura matemática de utilidad en la resolución de problemas. 


\section{Dialogia}

ALBARRÁN, Luis Miguel Iglesias; GÓMEZ, Isabel Pascual; ARTEAGA-MARTÍNEZ, Blanca. El aprendizaje del álgebra en Educación Secundaria: las estrategias metacognitivas desde la tecnología digital

Figura 6 - Muestra de trabajo de estudiante. Estructura en la resolución del problema

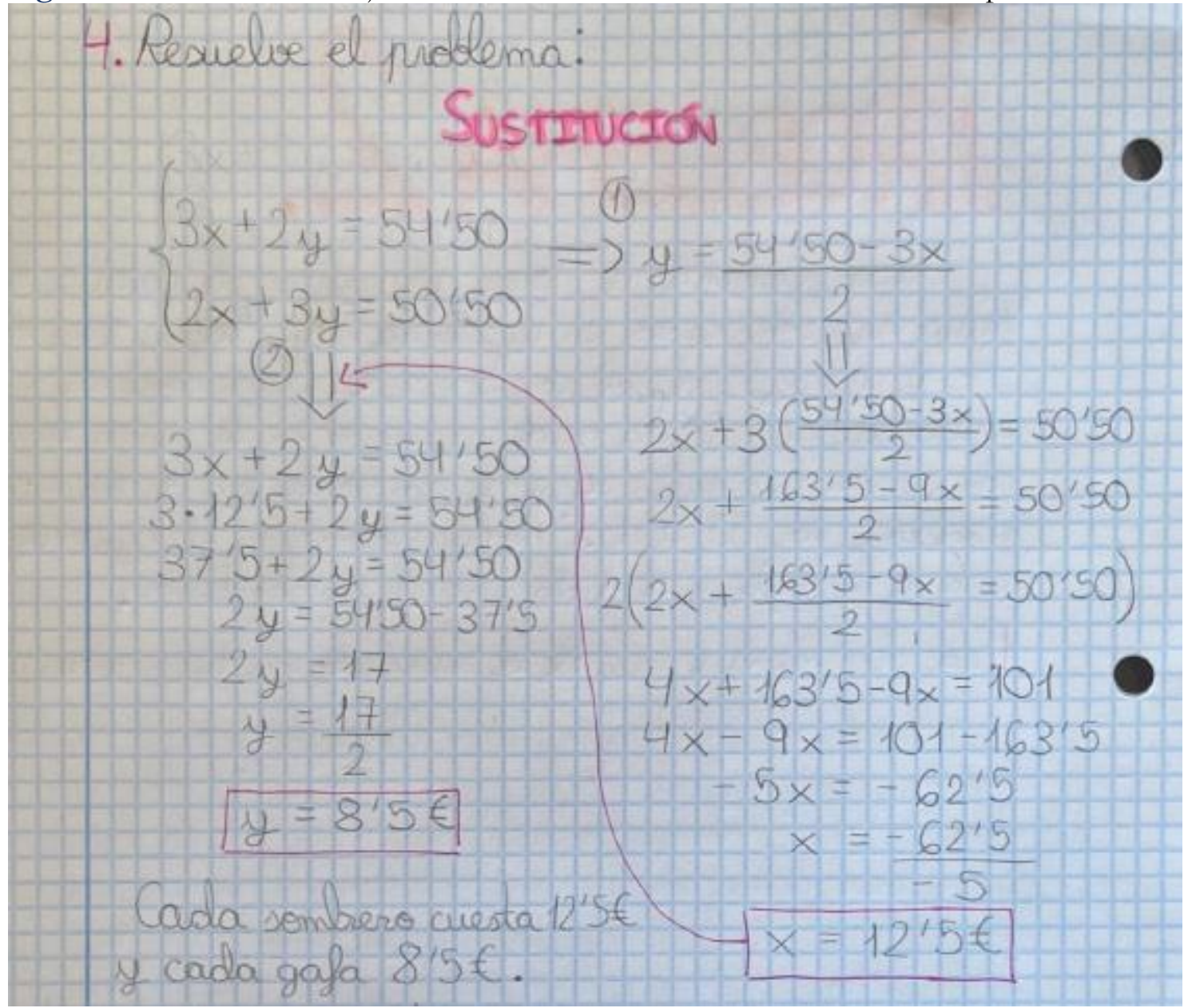

Fuente: Estudiante \#15.

El hecho de que se plantee al estudiante la reescritura del problema con sus palabras da lugar a un proceso de reflexión que facilita la traducción del lenguaje verbal al algebraico (Figura 7). 


\section{Dialogia}

ALBARRÁN, Luis Miguel Iglesias; GÓMEZ, Isabel Pascual; ARTEAGA-MARTÍNEZ, Blanca. El aprendizaje del álgebra en Educación Secundaria: las estrategias metacognitivas desde la tecnología digital

Figura 7 - Muestra de trabajo de estudiante. Traducción del lenguaje verbal al algebraico

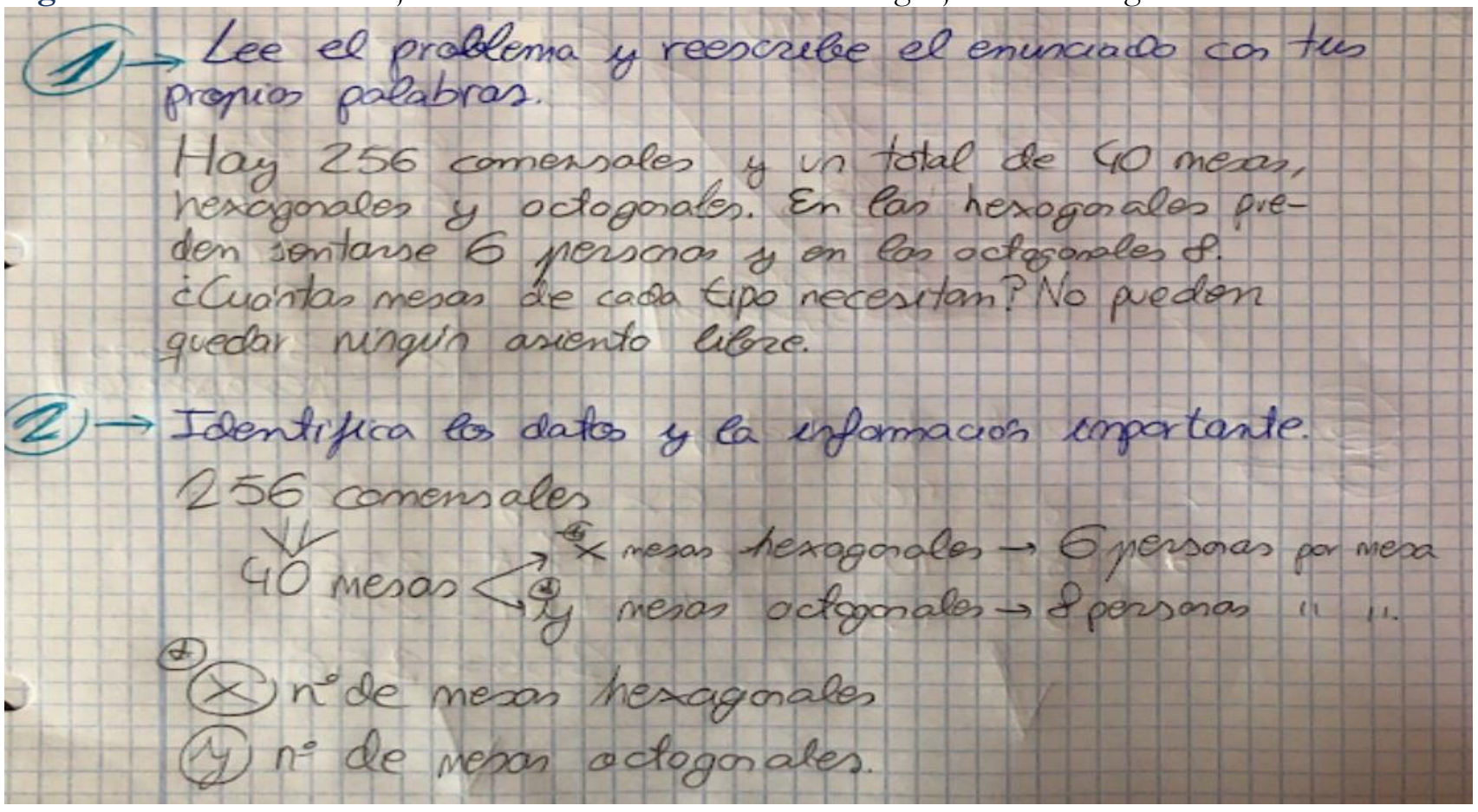

Fuente: Estudiante \#21.

Los estudiantes han adquirido un nivel óptimo en cuanto a la escritura matemática en formato digital como podemos ver en las muestras de trabajo (Figura 8). Hemos observado que al trabajar con la segunda plantilla, más guiada que la primera, se ha producido una reducción en la comunicación escrita. Si bien se han obtenido buenos resultados en la comprensión, representación visual y en la resolución del problema, tal vez sea debido a que los estudiantes han respondido mentalmente a las preguntas al mostrarse de manera explícita, han descuidado en muchos casos etiquetar y responder correctamente el problema. 
Figura 8 - Muestra de trabajo de estudiante. Estructura matemática en formato digital

\begin{tabular}{|c|}
\hline $\begin{array}{l}\text { Nombre y Apellidos: } \\
\text { Curso/Grupo: } 3^{\circ} \mathrm{D}\end{array}$ \\
\hline 4. Resuelve el problema \\
\hline $\begin{array}{l}\text { Pregúntate a ti mismo. (1) ¿Necesito fórmulas, ecuaciones o alguna notación especial? (2) ¿Sé ahora cómo } \\
\text { obtener la solución? }\end{array}$ \\
\hline $3 x+2 y=54,50 \quad(\times 3) \rightarrow 9 x+6 y=163,5$ \\
\hline \multirow{2}{*}{$\begin{aligned} 2 x+3 y=50,50 \quad(x-2) \rightarrow-4 x-6 y=-101 \\
5 x=62,5 \rightarrow x=52,5 / 5=12,5\end{aligned}$} \\
\hline \\
\hline $3 \times 12,5+2 y=54,50 \rightarrow 37,5+2 y=54,50 \rightarrow 2 y=54,50-37,5 \rightarrow 2 y=17 \rightarrow y=17 / 2=8,5$ \\
\hline 5. Comprueba tu trabajo \\
\hline Pregúntate a ti mismo. (1) ¿Los cálculos que he realizado son correctos? (2) ¿Es mi respuesta razonable? \\
\hline $3 \times 12,5+2 \times 8,5=54,50 \rightarrow 37,5+17=54,5 \rightarrow 54,5=54,5$ \\
\hline $2 \times 12,5+3 \times 8,5=50,50 \rightarrow 25+25,5=50,5 \rightarrow 50,5=50,5$ \\
\hline
\end{tabular}

Fuente: Estudiante \#13

\section{Conclusiones}

La competencia digital del docente a la hora de seleccionar y elaborar material para los estudiantes, junto al bagaje previo con los entornos digitales que poseían los estudiantes en un contexto de enseñanza tradicional, han posibilitado un desarrollo íntegro del currículo de álgebra en este grupo clase en un escenario de enseñanza a distancia. Es importante señalar este aspecto, ya que el grupo-clase no ha perdido el ritmo de trabajo, manteniendo un alto nivel de participación y alcanzando un nivel óptimo de aprendizaje, tal y como muestran los productos elaborados por los estudiantes y los logros evidenciados. Partiendo de un proceso de enseñanza-aprendizaje presencial, con materiales curriculares teóricos, se ha pasado a trabajar con materiales que ayudan a aprender de manera autónoma, con multitud de ejemplos resueltos paso a paso (retroalimentación inmediata), y con actividades exigentes y creativas, de alta demanda cognitiva. El grupo ha sido capaz de adaptarse a este escenario, apoyándose en el material metacognitivo -que ha favorecido que los estudiantes pudiesen desarrollar un aprendizaje autónomo- y en la rápida retroalimentación del docente -centrada en resolver los obstáculos que dificultaban la adquisición de los contenidos-

El apoyo del docente en situaciones de enseñanza a distancia en los niveles obligatorios es fundamental. Planificar las sesiones, realizando preguntas previas, estimula el pensamiento de los estudiantes y consigue mantener una demanda cognitiva de alto nivel durante la instrucción (BOALER; STAPLES, 2008). Este apoyo se materializa en la utilización de estrategias de 
comunicación variadas -tanto de carácter síncrono como asíncrono- y en la utilización de materiales que estimulen las estrategias reflexivas y el aprendizaje autónomo.

Cabe destacar la importancia que tiene en esta situación, donde el estudiante trabaja sin la supervisión en directo del docente, mantener el rigor en las tareas matemáticas. Según señalaron Stigler y Hiebert (2004) en su investigación analizando los vídeos de TIMMS, los estudiantes pasaban la mayor parte del tiempo realizando tareas de tipo procedimental, aunque lo programado por el docente estuviese considerado como de alta demanda cognitiva, y requiriese conexiones entre los contenidos.

Creemos de interés compartir parte de los materiales utilizados en esta investigación, en especial las plantillas de resolución de problemas y la clasificación en categorías, en aras de facilitar la replicabilidad de esta experiencia a cualquier entorno de aprendizaje (presencial, semi-presencial o en línea). Respecto a la comparación entre las dos plantillas, nuestra hipótesis de partida era que la utilización de la plantilla 2 , más guiada, iba a facilitar al estudiante tanto la comprensión como la expresión escrita de sus resultados de aprendizaje, sin embargo, hemos observado que los estudiantes hacen mayor trabajo mental pero plasman menos información en el papel. Estos resultados nos han hecho reflexionar. En un entorno digital, donde la observación de las tareas es el método fundamental de evaluación, quizás el tipo de instrucciones ofrecidas no deban ser demasiado concretas.

Utilizar distintos tipos de tareas conduce a distintos tipos de instrucción, y a distintas oportunidades de aprendizaje para los estudiantes (STEIN; SMITH; HENNINGSEN; SILVER, 2000), atendiendo a sus características individuales.

En un contexto de pandemia, escenario que exige mayor autonomía, hemos podido observar la capacidad de aprender a aprender de los estudiantes, un alto nivel de autorregulación en el aprendizaje, y resultados óptimos, tanto desde el punto de vista del rendimiento como del compromiso mostrado.

\section{Referencias}

ALLUEVA, P. Conceptos básicos sobre metacognición. En Desarrollo de habilidades metacognitivas: programa de intervención. Zaragoza: Consejería de Educación y Ciencia. Diputación General de Aragón, 2002. p. 59-85.

ALSINA, Á.; VÁSQUEZ ORTIZ, C. A. De la competencia matemática a la alfabetización probabilística en el aula: elementos para su caracterización y desarrollo. Unión: revista iberoamericana de educación matemática, n.48, p.41-58, 2016. 


\section{Dialogia}

ALBARRÁN, Luis Miguel Iglesias; GÓMEZ, Isabel Pascual; ARTEAGA-MARTÍNEZ, Blanca. El aprendizaje del álgebra en Educación Secundaria: las estrategias metacognitivas desde la tecnología digital

AREA, M.; ADELL, J. eLearning: Enseñar y aprender en espacios virtuales. En PABLOS, J. de (Coord). Tecnología Educativa. La formación del profesorado en la era de Internet. Málaga: Aljibe, 2009. p. 391-424.

ARTIGUE, M. Tecnologias de la información y de la comunicación y aprendizaje basado en la investigación: ¿Qué sinergias? En Las nuevas metodologías en la enseñanza y el aprendizaje de las Matemáticas. Segovia: Consejería de Educación de la Junta de Castilla y León, 2015. p. 17-28.

BOALER, J.; STAPLES, M. Creating mathematical futures through an equitable teaching approach: The case of Railside School. Teachers College Record, n.110, p. 608-645, 2008.

BOULTON-LEWIS, G. M.; COOPER, T. J.; ATWEH, B.; PILLAY, H.; WILLS, L. Readiness for algebra. In T. NAKAHARA; M. KOYAMA (Eds.). Proceedings of the 24th Conference for the International group for the psychology of mathematics education. Hiroshima, Japan: PME, 2001. p.89-96.

DORREGO, E. Educación a distancia y evaluación del aprendizaje. Revista De Educación a Distancia (RED), n. 50, p.1-20, 2016. Disponible en: http://dx.doi.org/10.6018/red/50/12

ESPAÑA [Andalucía]. Orden de 14 de julio de 2016. Currículo correspondiente a la Educación Secundaria Obligatoria en la Comunidad Autónoma de Andalucía. Boletín Oficial de la Junta de Andalucia, n.144, p.108-396, 2016.

FORD, D. J. The Effects of Metacognitive Training on Algebra Students' Calibration Accuracy, Achievement, and Mathematical Literacy. Dissertation, Teaching and Learning, Old Dominion University, 2018. Disponible en: https://digitalcommons.odu.edu/teachinglearning_etds/14

GAMA, C. A. Integrating metacognition instruction in interactive learning environments. (Tesis de doctorado). Brighton: University of Sussex, 2004. Disponible en:

http://homes.dcc.ufba.br/ claudiag/thesis/Thesis_Gama.pdf

IGLESIAS, L. M. Enseñanza y aprendizaje de las matemáticas en la era digital. Ambientes de aprendizaje mediados por TIC. En SCOPEO. e-MatemáTICas. Scopeo Monográfico, 4, 2012. p. 41-80. Disponible en: http://scopeo.usal.es/wp-content/uploads/2013/04/scopeom004.pdf

IGLESIAS, L. M. ¿Cómo de cerca a la perpendicular puedes llegar?, 2020a. Disponible en: https://www.geogebra.org/m/udtsbbeb

IGLESIAS, L. M. Laberinto de ecuaciones de primer y segundo grado, 2020b. Disponible en: https:/ / matematicas11235813.luismiglesias.es/laberinto-de-ecuaciones-de-primer-y-segundogrado-lmia-2/

KAPUT, J. Transforming Algebra from an Engine of Inequity to an Engine of Mathematical Power by "Algebrafying" the K-12 Curriculum. National Center for Improving Student Learning and Achievement in Mathematics and Science. Dartmouth, MA, 2010. Disponible en: https:// files.eric.ed.gov/fulltext/ED441664.pdf

LEE, YUJIN; CAPRARO, MARY M.; CAPRARO, ROBERT M.; BICER, ALI. A MetaAnalysis: Improvement of Students' Algebraic Reasoning through Metacognitive Training. International Education Studies, v.11, n.10, 2018. Disponible en: https:// files.eric.ed.gov/fulltext/EJ1192530.pdf 


\section{Dialogia}

ALBARRÁN, Luis Miguel Iglesias; GÓMEZ, Isabel Pascual; ARTEAGA-MARTÍNEZ, Blanca. El aprendizaje del álgebra en Educación Secundaria: las estrategias metacognitivas desde la tecnología digital

MALI, A.; GERAMI, S.; ULLAH, A.; MESA, V. Teacher Questioning in Problem Solving in Community College Algebra Classrooms. In Problem Solving in Mathematics Instruction and Teacher Professional Development. Cham: Springer, 2019. p. 317-335.

MARTINEZ, MARA V.; BRAGELMAN, JOHN; STOELINGA, TIMOTHY. Underprepared Students' Performance on Algebra in a Double-Period High School Mathematics Program. Mathematics Educator, v.25, n.1, p.3-31, 2016.

MECD. PIS A 2012 Informe español, 2012. Disponible en: http://www.mecd.gob.es/dctm/inee/internacional/pisa2012/pisa2012lineavolumeni.pd??docum entId=0901e $72 \mathrm{~b} 81786310$

MEVARECH, Z.; KRAMARSKI, B. Matemáticas críticas para las sociedades innovadoras: El papel de las pedagogías metacognitivas, México: OCDE, 2017. Disponible en:

https://doi.org/10.1787/9789264273078-es

NCTM, National Council of Teachers of Mathematics. Focus in high school mathematics: Reasoning and sense making. Reston, VA: AUTHOR, 2009.

PALM, T. Impact of authenticity on sense making in word problem solving. Educational Studies in Mathematics, n.67, p.37-58, 2008. Disponible en: https://doi.org/10.1007/s10649-007-9083-3.

RABINO, A.; CUELLO, P. Matemática Realista en la Educación Secundaria. Buenos Aires: Novedades Educativas, 2017.

SANTOS, L. M. Principios y métodos de la resolución de problemas en el aprendizaje de las matemáticas. México: Grupo Editorial Iberoamérica, 1996.

SANTOS, L. M. Resolución de problemas matemáticos: del trabajo de Pólya al razonamiento digital. En Rutas de la Educación Matemática. México: Sociedad Mexicana de Investigación y Divulgación de la Educación Matemática, 2018. p. 148-167.

SCHOENFELD, A. H. Learning to think mathematically: Problem solving, metacognition, and sense-making in mathematics. In Handbook for Research on Mathematics Teaching and Learning. New York: MacMillan, 1992. p. 334-370.

STEIN, M. K.; GROVER, B.; HENNINGSEN, M. Building student capacity for mathematical thinking and reasoning: An analysis of mathematical tasks used in reform classrooms. American Educational Research Journal, n.33, p. 455-488, 1996.

STEIN, M. K.; SMITH, M. S.; HENNINGSEN, M.; SILVER, E. A. Implementing standards-based mathematics instruction: A casebook for professional development. New York: Teachers, 2000.

STEUER, G.; ROSENTRITT-BRUNN, G.; DRESEL, M. Dealing with errors in mathematics classrooms: Structure and relevance of perceived error climate. Contemporary Educational Psychology, v.38, n.3, p.196-210, 2013. Disponible en: https://doi.org/10.1016/j.cedpsych.2013.03.002

STIGLER, J. W.; HIEBERT, J. Improving mathematics teaching. Educational Leadership, v.61, n.5, p.12-16, 2004. 


\section{Dialogia}

ALBARRÁN, Luis Miguel Iglesias; GÓMEZ, Isabel Pascual; ARTEAGA-MARTÍNEZ, Blanca. El aprendizaje del álgebra en Educación Secundaria: las estrategias metacognitivas desde la tecnología digital

TANIS, C. J. The seven principles of online learning: Feedback from faculty and alumni on its importance for teaching and learning. Research in Learning Technology, n.28, 2020. Disponible en: https://doi.org/10.25304/rlt.v28.2319.

Recebido em: 27 set. 2020/ Aprovado em: 19 nov. 2020

Cite como

(ABNT NBR 6023:2018)

ALBARRÁN, Luis Miguel Iglesias; GÓMEZ, Isabel Pascual; ARTEAGA-MARTÍNEZ, Blanca. El aprendizaje del álgebra en Educación Secundaria: las estrategias metacognitivas desde la tecnología digital.

Dialogia, São Paulo, n. 36, p. 49-72, set./dez. 2020. Disponível em: https:/ doi.org/10.5585/dialogia.n36.18279.

\section{American Psychological Association (APA)}

Albarrán, L. M. I., Gómez, I. P., \& Arteaga-Martínez, B. (2020, set./dez.). El aprendizaje del álgebra en Educación Secundaria: las estrategias metacognitivas desde la tecnología digital. Dialogia, São Paulo, 36, p. $49-72$.

https://doi.org/10.5585/dialogia.n36.18279. 